\title{
Efficacy of Bandage Contact Lenses versus Eye Patching in Early Postoperative Period of Müller's Muscle-Conjunctival Resection
}

\author{
Mehmet Serhat Mangan ${ }^{a}$ Hatice Tekcan ${ }^{a}$ Serap Yurttaser Ocak ${ }^{b}$ \\ aDivision of Ophthalmic Plastic and Reconstructive Surgery, Haydarpasa Numune Education and Research Hospital,

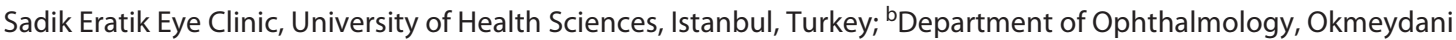 \\ Education and Research Hospital, University of Health Sciences, Istanbul, Turkey
}

\section{Keywords}

Müller's muscle-conjunctival resection · Bandage contact lens Eye patching $\cdot$ Patient comfort

\begin{abstract}
Purpose: Patients with blepharoptosis who are treated with Müller's muscle-conjunctival resection (MMCR) may experience postoperative pain and discomfort related to the suture material on the palpebral conjunctiva. This study aims to compare the postoperative subjective complaints, such as pain and discomfort, with a visual analog scale (VAS), and objectively evaluate the cornea with a tear break-up time (TBUT) test and fluorescein staining following MMCR that was managed by either a bandage contact lens $(B C L)$ or eye patching. Methods: Forty patients who had undergone a unilateral MMCR were randomized into 2 groups. Group 1 $(n=20)$ received a BCL, and group $2(n=20)$ received an eye patch following the MMCR. Patients were evaluated postoperatively at 1 and 7 days in this prospective study. The postoperative pain was measured using a VAS, and the requirement for anti-inflammatory medication was analyzed. A slitlamp examination was performed to evaluate the cornea with a TBUT test and fluorescein staining by objectively us-
\end{abstract}

ing the National Eye Institute (NEI) classification. Results: $\mathrm{BCL}$ treatment decreased the VAS score, the need for antiinflammatory medications, and fluorescein staining using the NEl score and increased the TBUT score significantly compared with the eye patch treatment $(p<0.001)$. Conclusions: Our findings suggest that the use of a $B C L$ may be beneficial in terms of reducing early postoperative pain and eye stinging, protecting the cornea, and retaining the ability to perform daily activities following a MMCR.

(C) 2020 S. Karger AG, Basel

\section{Introduction}

Müller's muscle-conjunctival resection (MMCR) procedure has recently become popular among surgeons [113]. This approach was first described by Putterman and Urist [2] in 1975. Possible problems regarding the MMCR ptosis treatment are associated with the presence of suture material on the palpebral conjunctiva. Complications may originate from the wound closure of this surgery that includes keratopathy, suture granuloma, infection, and the need for suture removal [3-5]. karger@karger.com

www.karger.com/ore

Karger $\stackrel{2}{=}$
(C) 2020 S. Karger AG, Basel
Mehmet Serhat Mangan

Department of Opthalmology, Haydarpasa Numune Education and Research Hospital Sadik Eratik Eye Clinic, University of Health Sciences

Tibbiye Cad. No. 23, Istanbul 34668 (Turkey)

mehmetsmangan@yahoo.com 


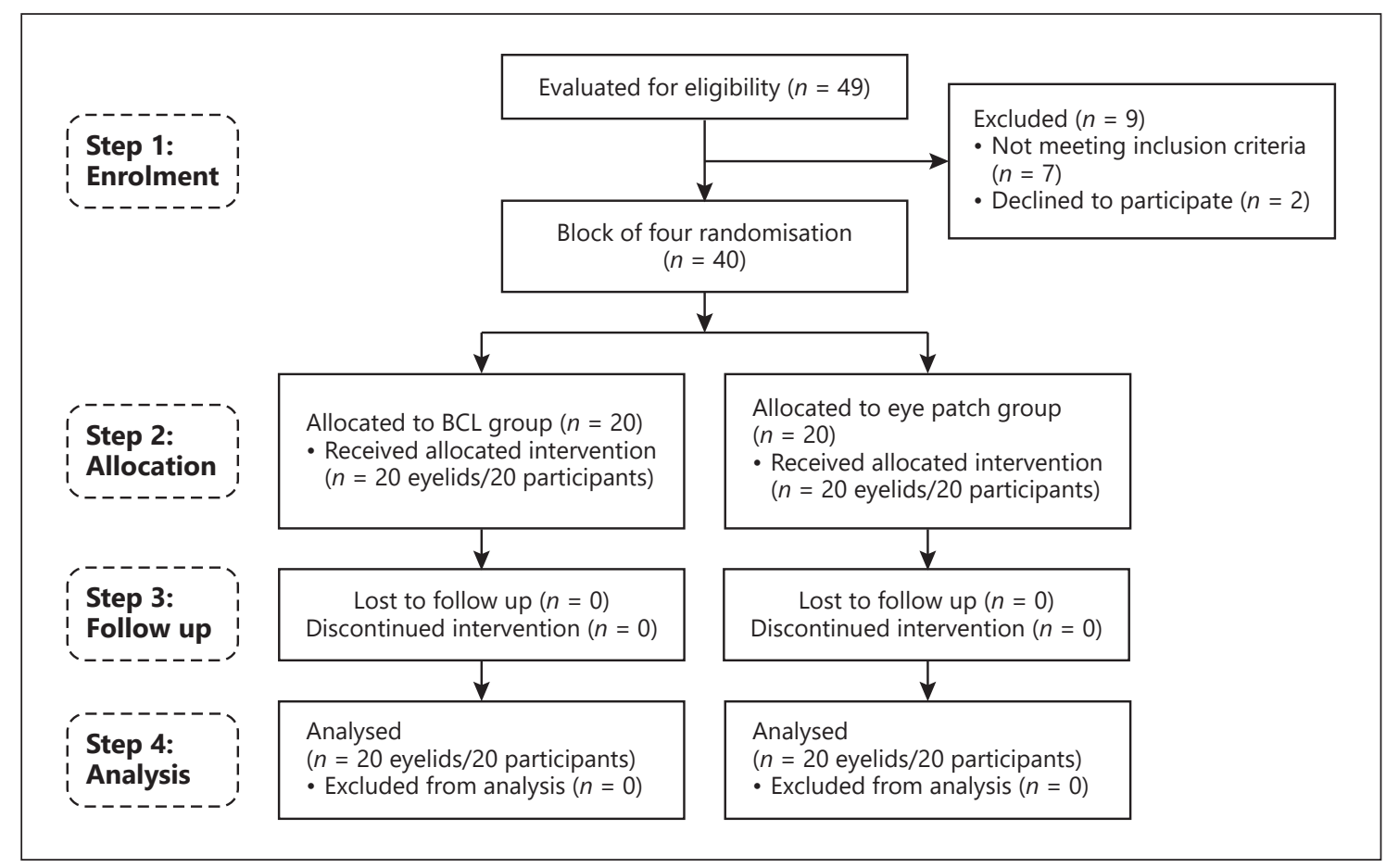

Fig. 1. Flowchart of the progress through the steps of a parallel randomized trial of the BCL and eye patching groups. BCL, bandage contact lens.

The problem of wound closure-related complications, such as postoperative pain and discomfort, may be significant and distressing for patients following a MMCR [3-5]. Perry et al. [3] reported that corneal abrasion was observed in $2 \%$ of eyelids after MMCR. Although significant keratopathy with the use of suture material is not common, many patients complain of suture-related discomfort [3]. A literature search revealed there is no standard treatment following MMCR and postoperative treatments are not usually noted in many published reports [8-10]. Systemic nonsteroidal anti-inflammatory drugs (NSAIDs), topical antibiotics, artificial tears, and/ or eye patching is commonly used to reduce the postoperative pain and provide protection to the cornea. However, eye patching results in preventing vision in the operated eye, often causes discomfort, disturbed blinking, and results in the patient refraining from social life for a period.

Different surgical modalities have been reported that reduce the risk of wound closure-related complications [3-6, 11-13]. Kavanagh et al. [4] reported that the MMCR ptosis repair using a fibrin sealant for wound closure is effective for postoperative pain and discomfort compared with sutures. In the literature, there are some studies con- cerning the use of a bandage contact lens (BCL) following pterygium surgery for improving postoperative patient comfort $[14,15]$. Therefore, we considered making use of the BCL following MMCR because it may improve the patient's comfort and protect the cornea in the early postoperative period. The aim of this study was to compare the postoperative subjective complaints, such as pain and discomfort, with a visual analog scale (VAS), and objectively evaluate the cornea with the tear break-up time (TBUT) test and fluorescein staining following MMCR that was managed by either a BCL or eye patching.

\section{Methods}

Study Design and Patient Selection

This prospective study included 40 patients (24 females and 16 males) who had unilateral ptosis and underwent unilateral MMCR on 1 eyelid performed from January 2017 to July 2019. This study was approved by the Research Ethics Committee of Hospital and was conducted in accordance with the tenets of the Declaration of Helsinki.

Participants were randomized into 2 groups by block-of-four randomizations. Group $1(n=20)$ received a BCL, while group 2 $(n=20)$ received eye patching. The allocation ratio was $1: 1$, and the randomization was done per participant, and participants with 
unilateral blepharoptosis would undergo the MMCR for more ptotic eyelid (Fig. 1). The surgeon was blinded to the type of treatment until the day of procedure. The exclusion criteria for the study were as follows: (1) a history of previous eyelid surgery (e.g., blepharoplasty and browpexy), (2) ocular surface disorders (e.g., ocular cicatricial pemphigoid and Stevens-Johnson syndrome), or (3) a short TBUT test ( $\leq 5 \mathrm{~s})$ or a corneal epitheliopathy by fluorescein staining.

\section{Surgical Procedure}

All patients underwent MMCR under local anesthesia. Preoperative or intraoperative sedation was not performed on any of the patients. Proparacaine hydrochloride $\left(0.5 \%\right.$ Alcaine $^{\circledR}$, SA AlconCouvreur, Puurs, Belgium) was topically administered on the eye before a sterile cover application with $10 \%$ povidone-iodine. $2 \%$ Lidocaine with 1:100,000 epinephrine was administered subconjunctivally and subcutaneously. The eyelid was everted by a Desmarres retractor. After the desired amount of resection was marked, the lid was grasped with a Putterman ptosis clamp and secured with 6-0 polypropylene in a continuous horizontal mattress pattern. The conjunctiva and Müller's muscle were excised, and the suture was externalized and tied over a bolster.

Study Groups

Group 1: A BCL was applied on the cornea at the end of the surgery, and the eye was not covered with a patch. Johnson \& Johnson Vision (Jacksonville, FL, USA), Acuvue Oasys with Hydraclear Plus (senofilcon A) BCL was used for all patients. Group 2: Antibiotic eye ointment (Tobramycin, $0.3 \%$ ) was applied on the eye, and the eye was covered with a patch at the end of the surgery.

\section{Postoperative Treatment}

Patients were discharged the same day after their surgery. Control visits of patients were done at 1 day and 1 week after surgery. The eye patch was removed 1 day after surgery. The contact lens and the suture were removed 1 week after surgery. The postoperative treatment regimen was the same for all patients. Loteprednol etabonate $(0.5 \%)$, tobramycin $(0.3 \%)$, ophthalmic suspension (Zylet ${ }^{\circledR}$, Bausch \& Lomb, Tampa, FL, USA), and artificial eye drops (sodium hyaluronate, Eyestil ${ }^{\circledR}$, Teka, Katania, Italy) were prescribed for a 15-day period to the patients.

\section{Evaluation Methods}

A VAS was used to measure postoperative subjective complaints, such as pain and discomfort, on the first and seventh postoperative days. Patients were asked to put a cross mark on a ruler of the $0-10$ scale expressing their score of subjective complaints ( 0 : lowest; 10: highest). The use and number of the anti-inflammatory medications were also analyzed.

A slit-lamp examination was performed on the first and seventh postoperative days to objectively evaluate the cornea with the TBUT test and fluorescein staining in a cobalt blue light. A single drop of $2 \%$ sodium fluorescein was instilled, and the TBUT was timed consecutively 3 times, and the average value was recorded. The corneal epitheliopathy was then assessed by fluorescein staining using the National Eye Institute (NEI) classification [16], where the area of corneal epitheliopathy was divided into 5 areas: superior, nasal, center, temporal, and inferior. The density of corneal epitheliopathy was graded from 0 , when no corneal epitheliopathy was present, to 1 when corneal epitheliopathy was sparse,
2 when it was moderately dense, and 3 when the corneal epitheliopathy was high and the lesions overlapped. Grade 0 was specified when no staining was present, and the maximum score was 15 . Each examination was performed by a masked observer.

The BCL was removed on the first and seventh postoperative days. The patients were examined by slit-lamp for evaluation of TBUT and corneal epitheliopathy an hour later when BCL was removed on that day in patients with group 1 . After the day 1 examination, a new BCL was reapplied to the eye until the seventh postoperative day.

\section{Statistical Analysis}

Statistical analyses were performed using SPSS (Statistical Package for Social Sciences) for Windows 25.0. The KolmogorovSmirnov test was used to check normal distributions of all parameters. Numerical variables are presented as a mean \pm standard deviation. For statistical analyses, the background data were analyzed using the Kruskal-Wallis test and the $\chi^{2}$ test to examine the overall balance among groups. If the Kruskal-Wallis test was statistically significant, the Student's $t$ test was performed, if not, the MannWhitney $U$ test was performed to assess which differences were significant. Statistical significance was defined as $p<0.05$.

\section{Results}

In total, 40 patients (24 females, 16 males) were enrolled in this study. The mean age of the patients in group 1 was $38.55 \pm 7.35$ years, while it was $38.9 \pm 7.29$ years in group 2 . There were no statistically significant differences between the 2 groups in terms of age, sex, or sample size. The follow-up period for patients in this study was 7 days. The distributions of mean scores on postoperative days 1 and 7, VAS scores, use of oral NSAIDs, NEI scores (total and 5 areas), and TBUT scores are presented in $\mathrm{Ta}$ ble 1 .

\section{Discussion}

Pain and discomfort are still issues following MMCR surgery. The manifestation of pain in the early postoperative period following MMCR is influenced by factors such as suture irritation, corneal epitheliopathy, and surgical trauma [3-5]. In MMCR, since the efficacy of subcutaneous and subconjunctival anesthesia continues for a short period, pain typically starts in the early postoperative period. Eye patching and topical antibiotic ointments are the most common treatments during the postoperative period $[7,17,18]$. If the pain is excessive, systemic NSAIDs can be used. However, patients with covered eyes feel uncomfortable, have no vision in the operated eye, and may refrain from social activities for a 
Table 1. The distributions of mean scores on postoperative days 1 and 7, for VAS scores, use of oral NSAIDs, NEI scores (total and 5 areas), and TBUT scores

\begin{tabular}{llll}
\hline & Group 1 & Group 2 & $\begin{array}{l}p \\
\text { value }\end{array}$ \\
& & & \\
\hline VAS (1st day) & $4.05 \pm 1.23$ & $6.20 \pm 1.01$ & 0.001 \\
VAS (7th day) & $1.90 \pm 0.79$ & $3.45 \pm 0.89$ & 0.001 \\
Use of oral NSAIDs, $n$ (1st day) & $0.70 \pm 0.47$ & $1.15 \pm 0.49$ & 0.01 \\
Use of oral NSAIDs, $n$ (7th day) & $0.10 \pm 0.31$ & $0.80 \pm 0.52$ & 0.001 \\
NEI score, total (1st day) & $3.50 \pm 1.15$ & $6.95 \pm 1.64$ & 0.001 \\
$\quad$ Superior & $1.15 \pm 0.37$ & $2.10 \pm 0.45$ & 0.001 \\
Nasal & $0.75 \pm 0.55$ & $1.60 \pm 0.50$ & 0.001 \\
Center & $0.80 \pm 0.41$ & $1.60 \pm 0.60$ & 0.001 \\
Temporal & $0.75 \pm 0.44$ & $1.45 \pm 0.60$ & 0.001 \\
Inferior & $0.05 \pm 0.22$ & $0.15 \pm 0.37$ & 0.3 \\
NEI score, total (7th day) & $1.50 \pm 0.69$ & $3.55 \pm 1.00$ & 0.001 \\
$\quad$ Superior & $1.00 \pm 0.00$ & $1.15 \pm 0.37$ & 0.001 \\
$\quad$ Nasal & $0.10 \pm 0.31$ & $0.90 \pm 0.31$ & 0.001 \\
Center & $0.30 \pm 0.47$ & $0.80 \pm 0.41$ & 0.001 \\
Temporal & $0.10 \pm 0.31$ & $0.70 \pm 0.47$ & 0.001 \\
Inferior & $0.00 \pm 0.00$ & $0.00 \pm 0.00$ & 0.3 \\
TBUT (1st day) & $9.22 \pm 0.93$ & $8.12 \pm 0.99$ & 0.001 \\
TBUT (7th day) & $9.78 \pm 0.95$ & $8.60 \pm 0.98$ & 0.001 \\
\hline
\end{tabular}

VAS, visual analog scale; NSAIDs, nonsteroidal anti-inflammatory drugs; NEI, National Eye Institute; TBUT, tear break-up time.

period. Therefore, we aimed to evaluate the efficacy of BCL treatments as an alternative to eye patching in this study. We compared the postoperative subjective complaints, such as pain and discomfort, and objectively evaluated the cornea with the TBUT test and fluorescein staining following MMCR in patients with BCL or eye patching.

There are many benefits to using a BCL. In the present study, we found that the BCL is more effective than eye patching following MMCR. This study revealed that VAS scores, NEI scores, and the use of oral NSAIDs on the postoperative first and seventh days were significantly reduced in the BCL group compared with the eye patching group. Moreover, in patients with the BCL, the TBUT score was increased compared to the eye patch group.

Kavanagh et al. [4] reported that a fibrin sealant used for wound closure during MMCR ptosis repair was as effective as the use of suture for wound closure, yet with fewer complications. These authors also noted that several patients in the suture group had suture-related complications, including suture granuloma, loose sutures, and suture-related keratopathy. However, none of the pa- tients in fibrin sealant group experienced such problems in the postoperative period. Nevertheless, there are some potential disadvantages in the use of the fibrin sealants. First, it is a human product, and there is a small but inherent risk of disease transmission. The second issue is that of cost, and fibrin sealant is contraindicated in patients with an aprotinin allergy, and its safety is unknown in pediatric or pregnant patients. These suture-related complications may be preventable. One alternative is a sutureless technique was reported by Lauring [6] in 1977. In recent years, sutureless technique has become popular again [11]. Additionally, Ediriwickrema et al. [12] reported the single suture MMCR modification and Carruth and Meyer [13] reported the simplified MMCR technique. In the present study, the BCL was not related to any extra complications to the cornea during the healing process and yielded good patient satisfaction. Additionally, Kavanagh et al. [4] reported that the suture techniques may vary in that both the conjunctiva and deeper tissues may be included during wound closure compared with the use of a fibrin sealant where wound closure is limited to the conjunctiva alone. These authors also noted that this difference was not reflected clinically in the results of the eyelid position and symmetry attained after ptosis repair.

In the present study, corneal parameters, such as NEI scores and TBUT scores, were better in group 1 patients compared to those in group 2 . These results are consistent with the Daglioglu et al. [14] study. BCL minimizes the suture-related keratopathy and protects the cornea. The explanation given by Daglioglu et al. [14] was that this issue might be related to an occasional uncovering of the cornea under the eye patch since the eye patch does not eliminate the eyelid movements [14]. However, it appears that this study compares using a contact lens for 1 week, compared to patching the eye for 1 day. These differences may have an influence on the NEI and TBUT scores between the 2 groups. Additionally, instillation of eye ointment versus a contact lens may lead to similar comfort. However, eye ointments are sticky and may cause blurred vision. Moreover, eye ointments contain preservatives, which represent a risk for the ocular surface [19]. Preservatives may disrupt the lipid layer of the tear film. This cannot be regenerated and can no longer protect the aqueous layer of the tear film, which evaporates easily. This effect may be associated with the impairment of the NEI and TBUT scores on the first postoperative day. La Porta Weber et al. [20] reported that contact lens acts as a protective covering for the cornea by controlling evaporation and maintaining direct contact between the fluid and the corneal epithelium. The contact lens also protects 
the cornea from abrasions and mechanical trauma, which commonly result from eyelid scar irregularities.

The BCL may reduce suture-related pain and discomfort following surgery by minimizing rubbing between the cornea and sutures. Patients' VAS scores for both the postoperative first day and first week were significantly lower in group 1 compared to group 2 in the present study. As a result, suture-associated pain, discomfort, and stinging were minimized. These results are consistent with the Daglioglu et al. [14] study. The explanation given by Daglioglu et al. [14] was that postoperative pain and discomfort are observed because of the contact between the cornea and eyelid sutures in eye patching. Therefore, the disturbed patient opens and closes the eye under the eye patch, which increases the pain. In addition, keeping the eye closed under the eye patch requires greater effort, which might cause fatigue of the periocular muscles [14].

Another important issue when considering the use of a BCL is the increased risk for infections. In the present study, we observed that using the BCL for 1 day and 1 week postoperatively may not be related to an infection risk. However, we prescribed antibiotic drops to all patients.

The use of the BCL for MMCR may work best in those patients with a history of the ocular surface disease, a previous corneal or refractive surgery, suture-associated problems, and underlying corneal erosions. An additional benefit of the BCL may be its use in monocular patients undergoing MMCR in their better, or only, eye. In these patients, the use of a BCL instead of an eye patch prevents visual problems and enables normal postoperative functioning and performance of daily activities.

This study has some limitations. The sample size was relatively small for the patients undergoing MMCR. In addition, the follow-up period was relatively short. In further studies, it would be helpful to include a long-term follow-up to determine if and when the eye patch group parameters (VAS, NEI, and TBUT score) were compared with the BCL group.
To our knowledge, this study is the first to use the BCL after MMCR and compare it with eye patching to relieve patients' postoperative pain and discomfort and protect the cornea. The findings of the present study suggest that the BCL may be beneficial in terms of reducing early postoperative pain and eye stinging, protecting the cornea, and maintaining daily activities following MMCR.

\section{Acknowledgement}

We thank Prof. Dr. Eray Yurtseven, for assistance with statistical analysis that improved the manuscript.

\section{Statement of Ethics}

This study was approved by the Research Ethics Committee of University of Health Sciences, Haydarpasa Numune Education and Research Hospital (the approval reference number: HNEAHKAEK 62977267-E.117) and was conducted in accordance with the tenets of the Declaration of Helsinki. Informed written consents were obtained from participants.

\section{Conflict of Interest Statement}

The authors have no conflicts of interest to declare.

\section{Funding Sources}

The authors did not receive any funding.

\section{Authors Contributions}

The manuscript has been read and approved for submission by all authors. All authors have contributed to preparing the manuscript in accordance with the International Committee of Medical Journal Editors (ICMJE) criteria for authorship. No person or persons other than the authors listed have contributed significantly to the preparation of the manuscript.

\section{References}

1 Putterman AM. Pearls for Muller's muscleconjunctival resection with upper blepharoplasty. In: Harstein ME, Massry GG, Holds JB, editors. Pearls and pitfalls in cosmetic oculoplastic surgery. 2nd ed. New York, NY: Springer; 2015. p. 655-7.

2 Putterman AM, Urist MJ. Müller muscleconjunctiva resection. Technique for treatment of blepharoptosis. Arch Ophthalmol. 1975;93(8):619-23.
3 Perry JD, Kadakia A, Foster JA. A new algorithm for ptosis repair using conjunctival Müllerectomy with or without tarsectomy. Ophthal Plast Reconstr Surg. 2002;18(6):426-9.

4 Kavanagh MC, Ohr MP, Czyz CN, Cahill KV, Perry JD, Holck DE, et al. Comparison of fibrin sealant versus suture for wound closure in Müller muscle-conjunctiva resection ptosis repair. Ophthal Plast Reconstr Surg. 2009; 25(2):99-102.
5 Czyz CN, Rich NE, Foster JA, Kavanagh MC, Perry JD, Holck DE. Comparison of postoperative eyelid position using fibrin sealant versus suture for wound closure in Müller's muscle-conjunctiva resection ptosis repair. Plast Reconstr Surg. 2011;128(2):423-30.

6 Lauring L. Blepharoptosis correction with the sutureless Fasanella-Servat operation. Arch Ophthalmol. 1977;95(4):671-4. 
7 Yang JW. Modified levator muscle resection using Putterman Muller's muscle-conjunctival resection-ptosis clamp. Aesthet Surg J. 2018;38(5):480-7.

8 Jang SY, Chin S, Jang JW. Ten years' experience with unilateral conjunctival mullerectomy in the Asian eyelid. Plast Reconstr Surg. 2014;133(4):879-86.

9 Choudhary MM, Chundury R, McNutt SA, Perry JD. Eyelid contour following conjunctival müllerectomy with or without tarsectomy blepharoptosis repair. Ophthal Plast Reconstr Surg. 2016;32(5):361-5.

10 Zauberman NA, Koval T, Kinori M, Matani A, Rosner M, Ben-Simon GJ. Müller's muscle-conjunctival resection for upper eyelid ptosis: correlation between amount of resected tissue and outcome. Br J Ophthalmol. 2013;97(4):408-11.

11 Gildener-Leapman JR, Sheps I, Stein R, Benyamini O, Milstein A, Hartstein ME. The sutureless mullerectomy. Ophthalmic Plast Reconstr Surg. 2019 May-Jun;35(3):290-3.
12 Ediriwickrema LS, Geng J, Nair AA, Prendes M, Gerber AL, Yang PT, et al. Single suture Müeller muscle conjunctival resection (ssMMCR): a modified technique for ptosis repair. Ophthalmic Plast Reconstr Surg. 2019; 35(4):403-6.

13 Carruth BP, Meyer DR. Simplified Müller's muscle-conjunctival resection internal ptosis repair. Ophthal Plast Reconstr Surg. 2013; 29(1):11-4.

14 Daglioglu MC, Coskun M, Ilhan N, Tuzcu EA, Ilhan O, Keskin U, et al. The effects of soft contact lens use on cornea and patient's recovery after autograft pterygium surgery. Cont Lens Anterior Eye. 2014;37(3):175-7.

15 Prat D, Zloto O, Ben Artsi E, Ben Simon GJ. Therapeutic contact lenses vs. tight bandage patching and pain following pterygium excision: a prospective randomized controlled study. Graefes Arch Clin Exp Ophthalmol. 2018;256(11):2143-8.
16 Lemp MA. Report of the national eye institute/industry workshop on clinical trials in dry eyes. CLAO J. 1995;21(4):221-32.

17 Zatezalo CC, Tavakoli M, Ayala-Haedo J, Callaway NF, Ko MJ, Anagnostopoulos AG, et al. A prospective randomized comparative clinical trial to analyze pain and surgical outcomes between frontal nerve blocks and subconjunctival anesthesia for conjunctival mullerectomy resection. Ophthalmic Plast Reconstr Surg. 2018 Nov-Dec;34(6):575-8.

18 Georgescu D, Cole E, Epstein G, Fountain T, Migliori M, Nguyen Q, et al. Müller muscleconjunctiva resection for blepharoptosis in patients with glaucoma filtering blebs. Ophthal Plast Reconstr Surg. 2007;23(4):285-7.

19 Furrer P, Mayer JM, Gurny R. Ocular tolerance of preservatives and alternatives. Eur J Pharm Biopharm. 2002;53(3):263-80.

20 La Porta Weber S, Becco de Souza R, Gomes JÁP, Hofling-Lima AL. The use of the Esclera scleral contact lens in the treatment of moderate to severe dry eye disease. Am J Ophthalmol. 2016;163:167-e1. 